\title{
Reliability of Stool Antigen Tests: Investigation of the Diagnostic Value of a New Immunochromatographic Helicobacter pylori Approach in Dyspeptic Patients
}

\author{
Huseyin Korkmaz ${ }^{1 *}$, Duygu Findik², Ceyha Ugurluoglu ${ }^{3}$, Yuksel Terzi ${ }^{4}$
}

\begin{abstract}
Background: A diagnosis of $\boldsymbol{H}$. pylori infection can be made by invasive or non-invasive methods. Several noninvasive diagnostic tests based on the detection of $\boldsymbol{H}$. pylori stool antigen (HpSA) have been developed. The Genx $H$. pylori stool antigen card test is a new rapid, non-invasive test that is based on monoclonal immunochromatographic assay. The aim of this study was to determine its sensitivity, specificity, and diagnostic accuracy for diagnosing $H$. pylori infection in adult patients. Materials and Methods: A total of 162 patients were included in the study. A gastric biopsy was collected for histopathology and rapid urease testing. Stool specimens for HpSA testing were also collected. Patients were considered $\boldsymbol{H}$.pylori positive if two invasive tests (histological and rapid urease tests) were positive. Results: Using the reference test, $50.6 \%$ of the samples were positive for $H$. pylori infection. The Genx $H$. pylori antigen test was positive in $19.7 \%$ of patients. The sensitivity, specificity, positive predictive value, negative predictive value, and diagnostic accuracy of the Genx $H$. pylori antigen test were $51.6 \%, 96.0 \%, 88.8 \%, 76.1 \%$, and $79.0 \%$, respectively. Conclusions: The Genx $H$.pylori stool antigen card test is a new non-invasive method that is fast and simple to perform but provides less reliable results.
\end{abstract}

Keywords: Helicobacter pylori - sensitivity - specificity - diagnostic accuracy - H. pylori antigen test

Asian Pac J Cancer Prev, 16 (2), 657-660

\section{Introduction}

Helicobacter pylori is one of the most common bacterial pathogens; it is found in half of the world's population (Lehours et al., 2007). This gram-negative bacterium, which colonizes the gastric epithelium, is recognized as the major cause of gastric carcinogenesis and other gastric diseases, such as gastritis, gastroduodenal ulcers, and lymphoproliferative disorders (Lehours et al., 2007; McColl 2010). Therefore, it is important to reliably detect $H$. pylori infection.

Since the discovery of $H$. pylori, several diagnostic methods have been developed to detect it. These methods can be divided into noninvasive methods-such as the urea breath test (UBT), serological tests, and H. pylori stool antigen (HpSA) tests-and invasive methods such as culture, histological examination, polymerase chain reaction (PCR), and the rapid urease test (RUT) (Vakil $\mathrm{N}$ et al., 2004; McNulty et al,. 2011). Because invasive tests require upper gastrointestinal endoscopy with gastric mucosa biopsies, there is substantial growing interest in noninvasive methods. However, the widespread use of noninvasive tests on a large population is restricted because noninvasive tests present several disadvantages.
Both the UBT and the HpSA tests can detect an active $H$. pylori infection; however, because the antibodies against H. pylori may remain present long after the pathogen is eradicated by therapy, serological testing does not distinguish between current and past infections (McNulty et al,. 2011; Korkmaz et al., 2013). Although the UBT is another very sensitive, specific, and diagnostically accurate method, it is expensive, and trained personal and complex instrumentation are required to obtain and read breath samples. Therefore, it seems that HpSA tests provide an alternative, simple, and noninvasive method to the UBT for the diagnosis of infection (Vakil et al,. 2004; Malfertheiner et al., 2007; McNulty et al,. 2011). The HpSA test was first introduced with a polyclonal antibody in 1997 (Korkmaz et al., 2013). Currently, many HpSA tests that use monoclonal antibodies are based on enzyme immunoassay (EIA) or immunochromatographic assay (ICA) and they are widely used to diagnose current $H$. pylori infection as an alternative to the invasive techniques (Calvet et al., 2010; Kesli et al., 2010; Ceken et al., 2011 ). However, few studies have compared the new monoclonal HpSA tests, and major differences in diagnostic accuracy have been observed (Calvet et al., 2010; Korkmaz et al., 2013; Shimoyama 2013).

${ }^{I}$ Department of Internal Medicine, Division of Gastroenterology, ${ }^{2}$ Department of Microbiology, ${ }^{3}$ Department of Pathology, Faculty of Medicine, Selcuk University, Selcuklu, ${ }^{4}$ Department of Statistics, Faculty of Science and Arts, Ondokuz Mayis University, Samsun, Turkey*For correspondence: husein68@hotmail.com 
The aim of this present study was to evaluate the efficacy of the new Genx H. pylori stool antigen test (commercial immunoassay kit, the $H$. pylori CARD test)-which is based on monoclonal ICA for the detection of $H$. pylori infection in dyspeptic patients-and find its sensitivity, specificity, negative and positive predictive values, and accuracy in the diagnosis of $H$.pylori infection.

\section{Materials and Methods}

\section{Patients}

This study consisted of 162 adult patients that presented with dyspeptic symptoms to the Gastroenterology Department of the Selcuk University Medical Faculty in Konya, Turkey for routine upper gastrointestinal endoscopy. The inclusion criteria of the patients were evaluated by the gastroenterologist. The exclusion criteria of the patients were as follows: age younger than 18, previous gastric surgery and/or $H$. pylori eradication treatment, recent use of bismuth-containing compounds or antibiotics (in the last 2 months) or proton pump inhibitors (in the last 4 weeks), long-term use of corticosteroids and/or immunosuppressants, pregnancy, and lactation. A history of bleeding and coagulation disorders and any contraindications for biopsy sampling were also included in the exclusion criteria. The data related to patients enrolled in the study were recorded using a questionnaire.

\section{Endoscopy and Biopsy Specimens}

Before the endoscopy, each patient signed an informed consent form. During the endoscopy, 2 antrum biopsies and 1 corpus biopsy for histological evaluation and 1 antrum biopsy and 1 corpus biopsy for the RUT were obtained from each patient. The RUT was performed using the manufacturer's instructions (Ballard Medical Products, Draper, UT), and the results were interpreted at 1 hour and 24 hours after sampling. The biopsy specimens were processed for histopathological examination according to the standard procedure. Hematoxylin-eosin staining and a special staining for $H$. pylori (i.e. Giemsa staining) were performed. All biopsy samples were examined by the same pathologist. If $H$. pylori infection was observed, the bacterial density was scored semi-quantitatively on an ordinal scale (ranging from 1 to 3 ) by the pathologist.

Stool Samples and Detection of H. pylori by Genx $H$. pylori Antigen Test

The patients provided fresh stool samples in airtight containers that were stored in a deep freezer $\left(-80^{\circ} \mathrm{C}\right)$ until the stool antigen tests were performed. The test device and sample were kept at room temperature $\left(15-30^{\circ} \mathrm{C}\right)$ prior to testing. Exclusion criteria of the stool samples were diarrhea, inadequate amount, and delayed delivery of the samples after collection.

The Genx H. pylori CARD Test (Genx Bioresearch GOSB Teknopark A.S. Gebze, Kocaeli, Turkey) is a rapid ICA test that uses a monoclonal anti-H. pylori antibody on a strip for the detection of $H$. pylori infections in

Table 1. Performance Data of the Genx H. pylori Stool Antigen Test for diagnosing H. pylori Infection Compared with Reference Method

\begin{tabular}{lc}
\hline & Genx H. pylori CARD Test \\
\hline Sensitivity (\%),(95\% CI) & $51.1 \%(34.02-69.20)$ \\
Specificity (\%),(95\% CI) & $95.0 \%(90.56-100.0)$ \\
Positive predictive value (PPV), (\%) & $91.3 \%$ \\
Negative predictive value (NPV), (\%) & $65.5 \%$ \\
Accuracy (\%) & $72.8 \%$ \\
Total positive (n) & 23 \\
False positive (n) & 2 \\
Total negative (n) & 58 \\
Falsenegative (n) & 20 \\
\hline
\end{tabular}

$* 95 \% \mathrm{CI}=95 \%$ confidence interval

Table 2. Published Results of EIA-based Stool Antigen Tests Used in Various Studies for the Diagnosis of $\boldsymbol{H}$. pylori Infection before Therapy

\begin{tabular}{|c|c|c|c|c|c|}
\hline Authors (Years) & Test & Patients & Gold Standard & $\begin{array}{c}\text { Sensitivity } \\
(\%)\end{array}$ & $\begin{array}{c}\text { Specificity } \\
(\%)\end{array}$ \\
\hline Chisholm et al. (2004) & I. Card STAT (M, ICT) & 87 & $\mathrm{C}, \mathrm{H}$ & 87.8 & 88.4 \\
\hline Trevisani et al. (2005) & I. Card STAT (M, ICT) & 105 & RUT, H & 85 & 93 \\
\hline Wu et al. (2006) & I. Card STAT (M, ICT) & 176 & RUT, H, C & 95.2 & 87 \\
\hline Kesli et al. (2010) & H. pylori Fecal Antigen Test (M, ICT) & 168 & RUT, H & 81.0 & 92 \\
\hline Calvet et al. (2010) & I. Card STAT (M, ICT) & 199 & RUT, H, UBT & 68.8 & 90 \\
\hline Ceken et al. (2011) & Helicobacter Antigen Quick Castte (M, ICT) & 100 & $\mathrm{H}$ & 68.9 & 100 \\
\hline \multirow[t]{8}{*}{ Ilktac et al. (2012) } & Helicobacter pylori Ag (M, ICT) & 91 & C, RUT, PCR & 95.5 & 92.6 \\
\hline & HEPYLORI (M, ICT) & 93 & C, RUT, PCR & 88.9 & 87.5 \\
\hline & Helicobacter Antigen Quick (M, ICT) & 100 & C, RUT, PCR & 87 & 87 \\
\hline & Helicobacter pylori Antigen Test(M, ICT) & 91 & C, RUT, PCR & 71.1 & 89.1 \\
\hline & H. pylori Blister Test (M, ICT) & 100 & C, RUT, PCR & 69.6 & 90.7 \\
\hline & Rapid Strip HpSA (M, ICT) & 100 & C, RUT, PCR & 65.2 & 96.3 \\
\hline & H. pylori (M, ICT) & 100 & C, RUT, PCR & 58.7 & 81.5 \\
\hline & H. pylori Ag (M, ICT) & 100 & C, RUT, PCR & 57.8 & 93.5 \\
\hline Jekarl et al (2013). & ASAN Easy Test H. pylori & 266 & H,PCR,S- EIA & 84.5 & 96.2 \\
\hline \multirow[t]{3}{*}{ Korkmaz et al (2013). } & I. Card STAT (M, ICT) & 198 & RUT, H & 68.9 & 92.6 \\
\hline & One-StepH.pylori antigen (M, ICT) & 198 & RUT, H & 86.7 & 88.9 \\
\hline & H. pylori Fecal antigen test (M, ICT) & 198 & RUT, H & 78.9 & 87.0 \\
\hline Osman et al. (2014) & Atlas Helicobacter pylori Antigen test & 59 & RUT & 91.7 & 100 \\
\hline
\end{tabular}

*ICT, immunochromatographic assay; S-EIA, stool antigen test by enzyme immunoassay; C, culture; H, histology; S, serology; RUT, rapid urease test; UBT, urea breath test; M; PCR, real-time polymerase chain reaction; monoclonal 
stool specimens. The test was performed according to the manufacturer's instructions. By using the applicator stick of the provided sample diluent vial, a small portion of stool specimen was transferred into the sample diluent and mixed well by shaking gently. The tip of the vial was broken off, and five drops $(150 \mu \mathrm{L})$ were added to the sample well in the test device. The test result was read after 10 minutes. A positive test result was indicated by the appearance of red band in the zone marked $\mathrm{C}$ (control line) and a red band in the zone marked $\mathrm{T}$ (result line). The sample was considered negative when only one red band appeared across the central window in the zone marked $\mathrm{C}$. If no colored bands appeared or only one band appeared in the $\mathrm{T}$ zone, the result was regarded as invalid, and if an inconclusive result was obtained, the test was repeated with a new strip.

H. pylori was defined as positive when both of the biopsy-based invasive test results-namely, the histopathology test and the RUT-were positive. The study was approved by the ethics committee of the Medical Faculty at Selcuk University, Konya, Turkey, and all patients gave their written informed consent for inclusion in the study.

\section{Statistical analysis}

Statistical analysis was performed with the chisquare test, and standard methods were used to calculate sensitivity, specificity, predictive values of positive and negative results, and $95 \%$ of the confidence intervals of these values. $\mathrm{p}<0.05$ was regarded as significant. Calculations were performed using conventional software (IBM SPSS Statistics Version 20.0, 1989, 2011. Chicago, USA).

\section{Results}

A total of 162 patients with dyspeptic symptoms were included in the study. Of these patients, 80/162 (49.4\%) were men, and $82 / 162(50.6 \%)$ were women. The mean (SD) age of the patients was $33.4 \pm 12.0$ years. The mean ages of male and female patients were $33.9 \pm 12.4$ and $31.1 \pm 11.6$, respectively.

The results of both invasive tests were combined and used as the reference test (histology plus RUT). Using this reference, $50.6 \%(82 / 162)$ of the samples were positive for $H$. pylori infection. The result showed that $42(52.5 \%)$ men and $40(48.8 \%)$ women were positive for $H$. pylori infection. There were no statistical differences between men and women in terms of $H$. pylori seropositivity (P> $0.05)$.

Of the samples, $46(28.3 \%)$ were positive by the Genx H.pylori CARD test. The sensitivity, specificity, positive and negative predictive values, and diagnostic accuracy of the test were $51.2 \%, 95.0 \%, 91.5 \%, 65.5 \%$, and $72.8 \%$, respectively (Table 1).

\section{Discussion}

The Maastricht III Consensus Report recommended a "test and treat" strategy in primary care settings, and the use of noninvasive testing has been recommended in adult patients under the age of 45 with persistent dyspepsia (Malfertheiner et al., 2007). In other words, noninvasive tests, which include the UBT, the HpSA test, and high accuracy serological kits, should be used for the diagnosis of H. pylori (Malfertheiner et al., 2007; Korkmaz et al., 2013).

The most important disadvantage of the serological tests that detect antibodies against $H$.pylori is the inability to distinguish between active infection and previous exposure to H. Pylori (Vakil et al., 2004; McNulty et al,. 2011). Therefore, the UBT and HpSA tests are the only noninvasive methods suitable for $H$. pylori infection eradication and control (Vakil et al., 2004). The major advantages of HpSA tests are ease of use, rapid result times, and reduced cost compared to the UBT (Kesli et al., 2010; Korkmaz et al., 2013; Shimoyama 2013 ). Thus, HpSA tests may be the only option for a noninvasive $H$. pylori diagnosis. The technical characteristics of HpSA tests are also important. ICA-based HpSA test results are available within minutes and do not require the use of laboratory equipment. These features of ICA tests represent clear advantages over EIAs, which must be performed in the laboratory and take more than two hours to complete (Calvet et al., 2010; Kesli et al, 2010 Korkmaz et al., 2013).

In this study we tested the new monoclonal ICA-based HpSA test. To the best of our knowledge, this is the first prospective study to examine the efficacy of the Genx $H$. pylori antigen test in the diagnosis of $H$. pylori infection. The most important finding of this study was that the new stool antigen test showed low sensitivity (51.2\%) and baseline diagnostic accuracy $(72.8 \%)$, resulting in a high number of false-negative test results (Table 1). Financial analysis of the tests is important, especially in developing countries such as Turkey. When the HpSA test used in our study is compared with UBT, the UBT is 10 times more expensive than the HpSA test.

The results of the ICA-based HpSA tests used in various studies for the diagnosis of $H$. pylori infection before therapy are presented in Table 2. After evaluating the results of other studies in Table 2, we concluded that there are major differences in the diagnostic accuracy of the different stool tests, even when monoclonal antibody based ICA tests are compared. They have shown variable results in the primary diagnosis, with sensitivities between $57.8 \%$ and $95.8 \%$ and specificities between $79.3 \%$ and $100 \%$.

Lower sensitivities for HpSA tests have occurred in special circumstances, such as those for patients undergoing proton pump inhibitors or bismuth therapy and for patients with liver cirrhosis or gastrointestinal bleeding (Calvet et al., 2002; Grino et al., 2003: Khalilpour et al., 2013). Although we excluded the patients undergoing proton pump inhibitor or bismuth therapy, a few patients with hidden gastrointestinal bleeding may have been included in the study, which may have resulted in the false-negative HpSA tests. Nonetheless, low colonization of bacteria in the stomach and the consequent low concentrations of $H$. pylori antigens in the feces could be sufficient to cause false-negative results (Korkmaz et al., 2013). False-positive reactions may result from 
Huseyin Korkmaz et al

other Helicobacter species, and this may have caused false-positive antigen test results (Haggerty et al., 2005; Valliani et al., 2013).

In conclusion, The Genx H.pylori stool antigen test is rapid, easy to use, and does not require expensive equipment. However, our results have shown that it has low agreement with the criterion standard and has provided less reliable results. For this reason, when only rapid HpSA diagnostic tests are used in the laboratory, it is very important to know the diagnostic accuracies of rapid tests and to evaluate the results according to the sensitivities and specificities of these tests. Reliable HpSA-ICA tests with high quality results may be useful for small laboratories and for primary care physicians that need to test for $H$. pylori infection in the office.

\section{References}

Ceken N, Yurtsever SG, Baran N, et al (2011). Comparison of Helicobacter pylori antibody detection in stool with other diagnostic tests for infection. Asian Pac J Cancer Prev, 12, 1077-81.

Calvet X, Sanfeliu I, Musulen E, et al (2002). Evaluation of Helicobacter pylori diagnostic methods in patients with liver cirrhosis. Aliment Pharmacol Ther, 16, 1283-9.

Calvet X, Lario S, Ramirez-Lazaro MJ, et al (2010). Comparative accuracy of 3 monoclonal stool tests for diagnosis of Helicobacter pylori infection among patients with dyspepsia. Clin Infect Dis, 50, 323-8.

Chisholm SA, Watson CL, Teare EL, Saverymuttu S, Owen RJ (2004). Non-invasive diagnosis of Helicobacter pylori infection in adult dyspeptic patients by stool antigen detection: does the rapid immunochromatography test provide a reliable alternative to conventional ELISA kits? J Med Microbiol, 53, 623-7.

Grino P, Pascual S, Such J, et al (2003). Comparison of stool immunoassay with standard methods for detection of Helicobacter pylori infection in patients with uppergastrointestinal bleeding of peptic origin. Eur J Gastroenterol Hepatol, 15, 525-9.

Haggerty TD, Perry S, Sanchez L, Perez-Perez G, Parsonnet J (2005). Significance of transiently positive enzyme-linked immunosorbent assay results in detection of Helicobacter pylori in stool samples from children. J Clin Microbiol, 43, 2220-3.

Ilktac M, Sahin A, Nazik N, Ongen B (2012). Immunochromatographic tests detecting Helicobacter pylori antigen in stool: comparison of the results of eight different commercial kits. ANKEM Derg, 26, 148-53 (in Turkish).

Jekarl DW, An YJ, Lee S, et al (2013). Evaluation of a newly developed rapid stool antigen test using an immunochromatographic assay to detect Helicobacter pylori. Jpn J Infect Dis, 66, 60-4.

Kesli R, Gokturk HS, Erbayrak M, Terzi Y(2010). Comparison of the diagnostic values of the 3 different stool antigen tests for the noninvasive diagnosis of Helicobacter pylori infection. J Investig Med, 58, 982-6.

Khalilpour A, Santhanam A, Wei LC, et al (2013). Antigenic proteins of Helicobacter pylori of potential diagnostic value. Asian Pac J Cancer Prev, 14, 1635-42.

Korkmaz H, Kesli R, Karabagli P, Terzi Y (2013). Comparasion of the diagnostic accuracy of five different stool antigen tests for the diagnosis of Helicobacter pylori infection. Helicobacter, 18, 384-91.

Lehours P, Yilmaz O (2007). Epidemiology of Helicobacter pylori infection. Helicobacter, 12, 1-3.

Malfertheiner P, Megraud F, O'Morain C, et al (2007). Current concepts in the management of Helicobacter pylori infection: the Maastricht III Consensus Report. Gut, 56, 772-81.

McColl KE (2010). Clinical practice. Helicobacter pyloriinfection. $N$ Engl J Med, 362, 1597-604

McNulty CA, Lehours P, Mégraud F (2011). Diagnosis of Helicobacter pylori infection. Helicobacter, 16, 10-18.

Osman HA, Hasan H, Suppian R, et al (2014). Evaluation of the Atlas Helicobacter pylori stool antigen test for diagnosis of infection in adult patients. Asian Pac J Cancer Prev, 15, 5245-7.

Shimoyama T (2013). Stool antigen tests for the management of Helicobacter pylori infection. World J Gastroenterol, 19, 8188-91.

Trevisani L, Sartori S, Rossi MR, et al (2005). Evaluation of a new rapid immunoassay for the detection of Helicobacter pylori in faeces: a prospective pilot study. Aliment Pharmacol Ther, 21, 485-9.

Vakil N, Vaira D (2004). Non-invasive tests for the diagnosis of H. pylori infection. Rev Gastroenterol Disord, 4, 1-6.

Valliani A, Khan F, Chagani B, et al (2013). Factors associated with Helicobacter pylori infection, results from a developing country - Pakistan. Asian Pac J Cancer Prev, 14, 53-6.

Wu DC, Wu IC, Wang SW, et al (2006). Comparison of stool enzyme immunoassay and immunochromatographic method for detecting Helicobacter pylori antigens before and after eradication. Diagn Microbiol Infect Dis, 56, 373-8 